\title{
Dufour and Soret effect on MHD convective fluid flow in a vertical porous plate with suction
}

\author{
${ }^{*}$ G. Balreddy ${ }^{1}$, M.N.Rajashekar ${ }^{2}$ \\ ${ }^{1}$ Mahatma Gandhi Institute of Technology, Hyderabad, India \\ ${ }^{2}$ JNTUH College of Engineering Nachupally, Karimnagar, India \\ Corresponding Author: G. Balreddy
}

\begin{abstract}
The present paper is aimed at numerical investigation on the problem of heat and mass transfer on MHD boundary layer with chemical reaction, suction, Soret and Dufour effects. The properties of the fluid considered in present numerical study have been assumed to constant and the variation of density with buoyancy force is also analysed. The governing equations of the flow are transformed into non-linear ordinary differential equations and then solved by using Keller-box method. The various parameters like Magnetic parameter (M), thermal Grashof number (Gr), mass Grashof number $(\mathrm{Gc})$, permeability parameter $(\mathrm{K})$, Prandtl number $(\mathrm{Pr})$, Dufour number $(\mathrm{Du})$, Soret number $(\mathrm{Sr})$ on the flow are analyzed and shown in graphs. The numerical values of the Skin friction coefficient, Nusselt number and the Sherwood number are tabulated.
\end{abstract}

Keywords: Killer box Method, MHD fluid flow, Heat and Mass transfer, Soret effect, Suction

\section{INTRODUCTION}

Natural convection has attracted a great deal of attention from researchers because of its presence both in nature and engineering applications. In engineering applications, convection is commonly visualized in the formation of microstructures during the cooling of molten metals, and fluid flows around shrouded heat dissipation fins, and solar ponds. Soret and Dufour effects are interesting physical phenomenon in fluid mechanics, when heat and mass transfer occur simultaneously, the relations between the fluxes and the driving potentials are of a more intricate nature. It has been found that energy flux can be generated not only by temperature gradients but also by concentration gradients. The heat transfer caused by a concentration gradient is termed as diffusion thermo (Dufour) effect. On the other hand, mass transfer created by temperature gradients is called as thermal - diffusion (Soret) effect. Generally, in heat transfer process, the Soret and Dufour effects are neglected because they are smaller order of magnitude than the effects described by Fouriers and Ficks laws. The Soret effect has been utilized for isotope separation and in mixture between gases of very light molecular weight and of medium molecular weight. The importance of these effects in convective transport has been reported by Eckert and Drake (1972). Magnetohydrodynamic (MHD) flows have many applications in solar physics, geophysics, cosmic fluid dynamics, chemical engineering and electronics. Huges and Young (1996) gave an excellent summary of applications. In recent years, the flows of fluid through porous media have application in many branches of science and technology, viz., in the field of agriculture engineering tostudy the underground water resources, seepage of water in river-beds, in petroleum technology to study the movement of natural gas, oil and water through oil reservoirs, in chemical engineering for filtration and purification processes. Raptis et al. (1982) discussed the problem of hydromagnetic free convection flow through a porous medium between two parallel plates. The heat and mass transfer effect on the free convection boundary layer flow in a porous medium studied by Bejan and Khair (1985). The heat transfer in the thermal boundary layer of magneto-hydrodynamic flow over a flat plate analyzed by Watanabe and Pop (1994). The influence of thermal dispersion and stratification on non-darcy mixed convection flow from a vertical plate embedded in a porous medium has been investigated by Hassanien et al. (1998). Combined heat and mass transfer by mixed convection flow over a vertical plate embedded in a uniform porous medium in the presence of thermal dispersion has been investigated by Chamkha and Khaled (2000). Sattar et al. (2001) investigated the similar solutions for free convection and mass transfer flow in the presence of magnetic parameter. Muthucumaraswamy (2002) presented heat and mass transfer effects on a continuously moving isothermal vertical surface with uniform suction by considering 
chemical reaction. Chamkha (2003) have reported that the chemical reaction effects on MHD flow of uniformly stretched vertical permeable surface with heat generation/absorption. Cookey et al. (2003) investigated the MHD steady free convection flow past a infinite heated vertical plate in porous medium under the influence of viscous dissipation, radiation, time dependent suction. Abd El-Naby et al. (2004) examined the effect of radiation on MHD unsteady free convection flow over a vertical porous plate by using implicit finite difference method. Rahman and Sattar (2006) studied the effect of magnetic field on convective flow of a micropolar fluid past a continuously moving vertical porous plate in presence of heat generation or absorption. Ibrahim et al. (2008) considered the effect of chemical reaction, heat source and suction on the unsteady MHD free convection flow past a semi-infinite vertical permeable moving plate. Mansour et al. (2008) presented the influence of chemical reaction and viscous dissipation on MHD natural convection flow. Ibrahim et al. (2010) studied the MHD boundary layer flow of heat and mass transfer over a moving vertical plate. The effects of chemical reaction and radiation absorption on an unsteady MHD convective heat and mass transfer flow past a semi - infinite vertical moving in a porous medium was analyzed by Seethamahalakshmi et al. (2011). Sugunamma et al. (2011) presented the radiation effects on natural convection with magnetic field between heated inclined plates. Uwanta (2012) studied the effects of chemical reaction and radiation on heat and mass transfer past a semi-infinite vertical porous plate in the presence of constant mass flux and dissipation. Vidyasagar et al. (2013) consider the effects of heat and mass transfer over a moving vertical porous plate.

The focuss of the attempt is to model the Dufour and Soret effects on MHD boundary layer flow of a moving vertical porous plate with suction. The governing equations of the flow are coupled and solved numerically using by Killer box method. The effects of various emerging parameters on the velocity and temperature are modeled in present numerical attempt. The results of the analysis are presented in graphs.

\section{MATHEMATICAL FORMULATION}

Considering a two-dimensional steady boundary layer flow of a viscous incompressible electrically conducting fluid past a linearly started porous semi-infinite vertical plate embedded in a porous medium with Soret and Dufour effects. Here $x$-axis is taken along the direction of plate and $y$-axis is normal to it. All the fluid properties are assumed to be constant except for the density variations causing the buoyancy force represented by the body force term in the momentum equation. The continuity, momentum, energy and concentration equations describing the flow under the Boussinesq approximation can be written as

$\frac{\partial u}{\partial x}+\frac{\partial v}{\partial y}=0$

$u \frac{\partial u}{\partial x}+v \frac{\partial v}{\partial y}=v \frac{\partial^{2} u}{\partial y^{2}}+\beta g\left(T-T_{\infty}\right)+\beta^{*} g\left(C-C_{\infty}\right)-$ $\frac{\sigma}{\rho} \beta_{0}^{2} u-\frac{\vartheta}{K^{\prime}} u$

$u \frac{\partial T}{\partial x}+v \frac{\partial T}{\partial y}=\propto \frac{\partial^{2} T}{\partial y^{2}}+\frac{D_{m}}{C_{s}} \frac{K_{T}}{C_{P}} \frac{\partial^{2} C}{\partial y^{2}}$

$u \frac{\partial C}{\partial x}+v \frac{\partial C}{\partial y}=D_{m} \frac{\partial^{2} c}{\partial y^{2}}+\frac{D_{m}}{T_{m}} k_{T} \frac{\partial^{2} T}{\partial y^{2}}-K\left(C-C_{\infty}\right)$

(4)

where $\mathrm{u}$ and $\mathrm{v}$ are the velocity components in $x$ and $y$ directions, $\mathrm{T}$ is the temperature of the fluid, $\mathrm{C}$ is the species concentration in the boundary layer, $\vartheta$ is the kinematics viscosity, $\mathrm{g}$ is the acceleration due to gravity, $\beta$ is the coefficient of thermal expansion, $\beta^{*}$ is the coefficient of solutal expansion, $T_{\infty}$ is the temperature for away from the plate, $C_{\infty}$ Species concentration of the ambient fluid, $\sigma$ is the electric conductivity, $B_{0}$ is the uniform magnetic field, $\rho$ is the density, $K^{\prime}$ is the permeability, $\alpha$ is the thermal diffusivity, Dm is the mass diffusivity, $K_{T}$ is the thermal diffusion ratio and $C_{S}$ is the concentration susceptibility, $C_{P}$ is the specific heat at constant pressure, $T_{m}$ is the mean fluid temperature.

The boundary conditions of the problem are:

$\mathrm{u}=\mathrm{bx}, \mathrm{v}=\mathrm{V}, \mathrm{T}=\mathrm{Tw}=T_{\infty}+\mathrm{ax}, \mathrm{C}=C_{\infty}+\mathrm{bx}$

When $\mathrm{y}=0$

$u \rightarrow 0, T \rightarrow T_{\infty}, C \rightarrow C_{\infty}$, as $y \rightarrow \infty$

Temperature and concentration profiles, $V$ are the suction velocity at the plate surface.

In order to where B is a constant, a and b denotes the stratification rate of the gradient of ambient obtain similarity solutions of the problem, the following similarity variables are introduced

$$
\begin{aligned}
& \eta=\sqrt{\frac{B}{\vartheta}} y, F(\eta)=\frac{\psi}{x \sqrt{B \vartheta}}, \theta(\eta)=\frac{T-T_{\infty}}{T_{W}-T_{\infty}}, \\
& \phi(\eta)=\frac{C-C_{\infty}}{C_{W}-C_{\infty}}, F_{W}=\frac{V}{\sqrt{B \vartheta}}
\end{aligned}
$$

Where $\psi$ is the physical stream function defined as $u=\frac{\partial \psi}{\partial y}, v=-\frac{\partial \psi}{\partial x}$, so that the continuity equation (1) is automatically satisfied, $F(\eta)$ is the dimensionless stream function, $\theta$ is the nondimensional temperature function, $\phi$ is the nondimensional concentration, fw is the non-dimensional suction velocity and $\eta$ is the similarity variable. 
Introducing the relation (6) into the equations

(2)-(4), we obtain

$F^{\prime \prime \prime}+F F^{\prime \prime}-\left(F^{\prime}\right)^{2}+G_{r} \theta+G_{C} \emptyset-(M+K) F^{\prime}=0$

$\theta^{\prime \prime}+P_{r}\left(F \theta^{\prime}-F^{\prime} \theta\right)+D u P_{r} \emptyset^{\prime \prime}=0$

$\emptyset^{\prime \prime}+S_{C}\left(F \emptyset^{\prime}-F^{\prime} \emptyset\right)+S_{r} S_{C} \theta^{\prime \prime}-S_{C} K_{r} \emptyset=0$

(7.3)

where the primes indicate the differentiation with respect to $\eta, G_{r}=\frac{g \beta\left(T_{W}-T_{\infty}\right)}{x B^{2}}$, is the local temperature Grashof number, $G_{c}=\frac{g \beta^{*}\left(C_{W}-C_{\infty}\right)}{x B^{2}}$ is the local concentration Grashof number, $M=\frac{\sigma \beta^{2}}{\rho B} \quad$ is the magnetic parameter, $K=\frac{\vartheta}{K^{\prime} B} \quad$ is the permeability parameter, $P_{r}=\frac{\vartheta}{\alpha} \quad$ is the Prandtl number, $D_{u}=$ $\frac{D_{m} K_{T}\left(T_{W}-T_{\infty}\right)}{C_{S} C_{P} \vartheta\left(C_{W}-C_{\infty}\right)} \quad$ is the Dufour number, $S_{r}=$ $\frac{D_{m} K_{T}\left(T_{W}-T_{\infty}\right)}{T_{m} \vartheta\left(C_{W}-C_{\infty}\right)}$ is the Soret number and $S_{C}=\frac{\vartheta}{D} \quad$ is the Schmidt number

The corresponding boundary conditions are (8.1)

$$
F=-F_{W}, F^{\prime}=1, \theta=1, \emptyset=1 \text { when } \eta=0
$$

(8.2)-

The quantities of main physical interest are the skin friction coefficient (rate of shear stress), the Nusselt number (rate of heat transfer) and the Sherwood number (rate of mass transfer) which are proportional to $F^{\prime \prime}(0),-\theta^{\prime}(0)$ and $-\emptyset^{\prime}(0)$

\section{RESULTS AND DISCUSSION}

The governing equations $(7 \mathrm{a}-7 \mathrm{c})$ are steady, coupled and non-linear with initial and boundary conditions $(8 \mathrm{a}, 8 \mathrm{~b})$. They are solved numerically by using killer box method. For the purpose of discussing the results, the numerical calculations are presented in the form of non-dimensional velocity, temperature and concentration profiles. Numerical computations have been carried out for different values of $\mathrm{Gr}, \mathrm{Gc}, \mathrm{M}, \mathrm{K}, \mathrm{Pr}, \mathrm{Du}, \mathrm{Sc}, \mathrm{Sr}$. To demonstrate successful implementation of the numerical scheme, the numerical results are compared by a previous published work of Ibrahim and Makinde (2010) for skin friction coefficient, Sherwood number and Nusselt numbers in table 1 in the absence of Permeability Parameter K, Dufour number Du, Soret number Sr. The results are found to be in excellent agreement. Finally, the influence the flow parameters such as M, K, Pr, Du, Sr, fw on Skin friction coefficient , $K_{r}$, Nusselt number and Sherwood Number are highlighted in Table 2. The behaviour of these parameters is self-evident from the Table 2 and hence they will not discuss any further due to brevity.

Table 1: Computations showing comparison of $F^{\prime \prime}(0),-\theta^{\prime}(0)$ and $-\emptyset^{\prime}(0)$

When $\mathrm{Pr}=0.71, \mathrm{Du}=\mathrm{Sr}=\mathrm{K}=0$ with Ibrahim et al. (2010)

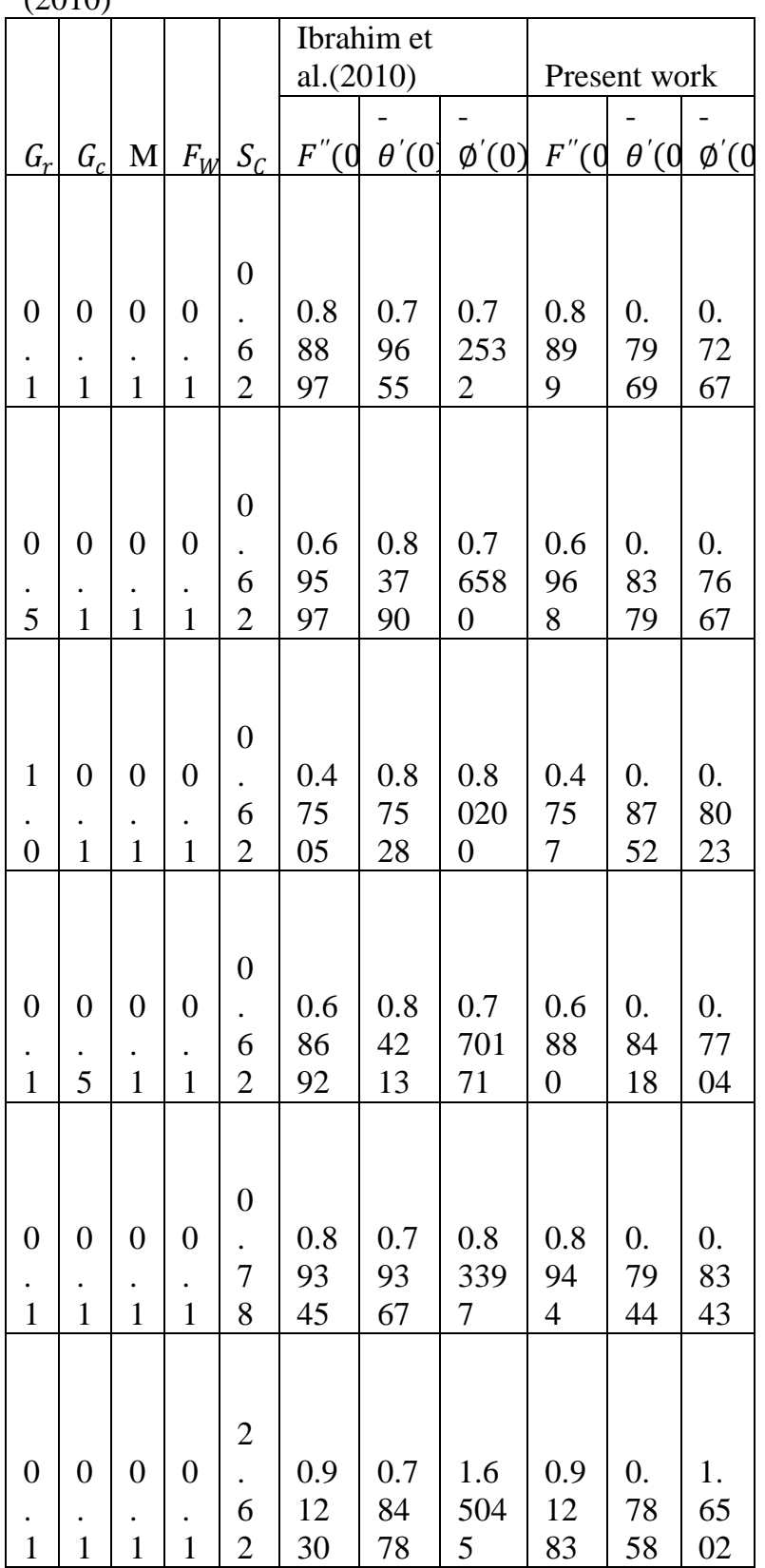


Table 2: $F^{\prime \prime}(0),-\theta^{\prime}(0)$ and $-\emptyset^{\prime}(0)$ for different values of $\mathrm{K}, \mathrm{M}, K_{r}, \mathrm{Pr}, \mathrm{Du}, \mathrm{Sr}, \mathrm{Sc}, F_{W}$

When $\mathrm{Gr}=0.1, \mathrm{Gc}=0.1$

\begin{tabular}{|l|l|l|l|l|l|l|l|l|l|l|}
\hline & & & & $\mathrm{D}$ & $\mathrm{S}$ & & & & - & - \\
$\mathrm{M}$ & $K$ & $K_{r}$ & $\mathrm{Pr}$ & $\mathrm{u}$ & $\mathrm{r}$ & $\mathrm{Sc}$ & $F_{W}$ & $F^{\prime \prime}(0$ & $\theta^{\prime}(0)$ & $\emptyset^{\prime}(0$ \\
\hline 0 & 0 & 0 & & 0 & 1 & & & & & \\
. &. &. & 0. &. &. & 0. & 0. & 1.1 & 0.7 & \\
1 & 1 & 5 & 72 & 1 & 0 & 22 & 1 & 726 & 920 & 1 \\
\hline $\mathbf{0}$ & 0 & 0 & & 0 & 1 & & & & & \\
. &. &. & 0. &. &. & 0. & 0. & 1.3 & 0.7 & \\
$\mathbf{5}$ & 1 & 0 & 72 & 1 & 0 & 22 & 1 & 541 & 401 & 1 \\
\hline 0 & $\mathbf{1}$ & 0 & & 0 & 1 & & & & & \\
. & $\cdot$ &. & 0. &. &. & 0. & 0. & 1.5 & 0.7 & \\
1 & $\mathbf{0}$ & 8 & 72 & 1 & 0 & 22 & 1 & 252 & 019 & 1 \\
\hline 0 & 0 & 1 & & 0 & 1 & & & & & \\
. &. &. & $\mathbf{1 .}$ &. &. & 0. & 0. & 1.2 & 0.9 & \\
1 & 1 & 6 & $\mathbf{0}$ & 1 & 0 & 22 & 1 & 276 & 602 & 1 \\
\hline
\end{tabular}

The Fig (1a), (1b) and (1c) show the profile of themal impact on on the dimensionless velocity, temperature and concentration, It is found in the study that an increase in $\mathrm{Gr}$ enhances the value of velocity. The temperature difference $(\mathrm{Tw}-\mathrm{T} \infty)$ is found to increase with Grashof number (Gr). Therefore an increase in the velocity due to the enhanced convection is caused. From Fig (1b), it is observed that the effect of increasing the values of $\mathrm{Gr}$ causes the reduction in the temperature profiles. It is interesting to note that the concentration profiles decrease as the Grashof number increases as indicated in study Fig (1c). The presented graphs clearly convey that the effect of Grashof number (Gr) causes the decrease in the concentration distribution as the concentration distribution species is dispersed away largely because of an increased temperature gradient. It is observes in the present that Mass Grashof Number $(\mathrm{Gc})$ has the same effect as $\mathrm{Gr}$ on the flow properties as shown in Figs $(2 \mathrm{a}-2 \mathrm{c})$

Figs (3a-3c) represent the influence of the Magnetic Parameter $\mathbf{M}$ on the velocity, temperature and concentration. The results obtained in the study as in figures revealthat the velocity will decrease and temperature profiles increase with the magnetic parameter. It is noted that a small change of increase of magnetic parameter causes the increase in concentration of the fluid. The presence of the magnetic field reduces the velocity profiles in the boundary layer region due to the induced force and the Lorentz force, caused by the magnetic field.

The variation of Prandtl number (Pr) on the temperature is shown in Fig 4. The thermal boundary layer thickness decrease with in increase in Prandtl number.For the different values of the permeability parameter $(\mathrm{K})$, the profiles of the velocity across the boundary layer are shown in Fig 5. The increase in the permeability parameter $\mathrm{K}$ makes the velocity reduce.
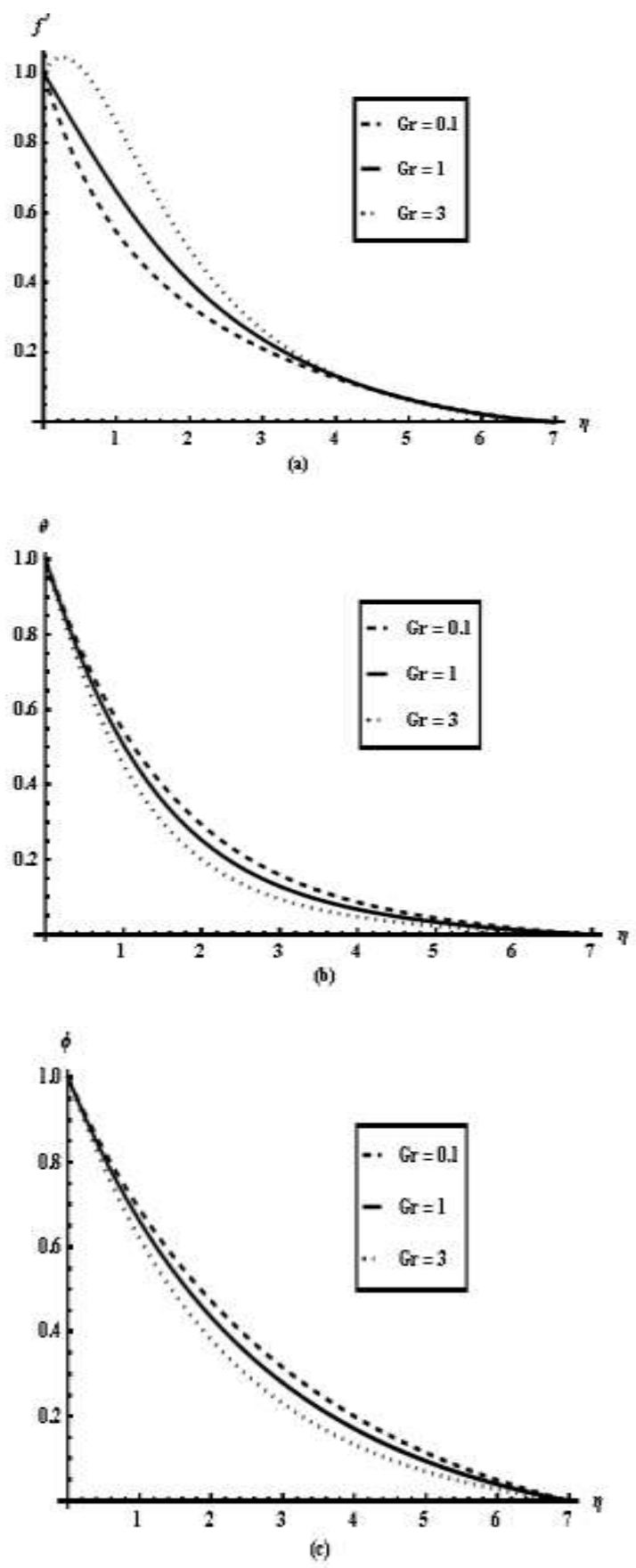

Fig 1. Effect of Gr on (a) Velocity, (b) Temperature and (c)Concentration profiles with $\mathrm{M}=1, \mathrm{~K}=0.1, \operatorname{Pr}=0.71, \mathrm{Du}=1, \mathrm{Sc}=0.22$, $\mathrm{Sr}=0.1, \mathrm{fw}=0.5$ 

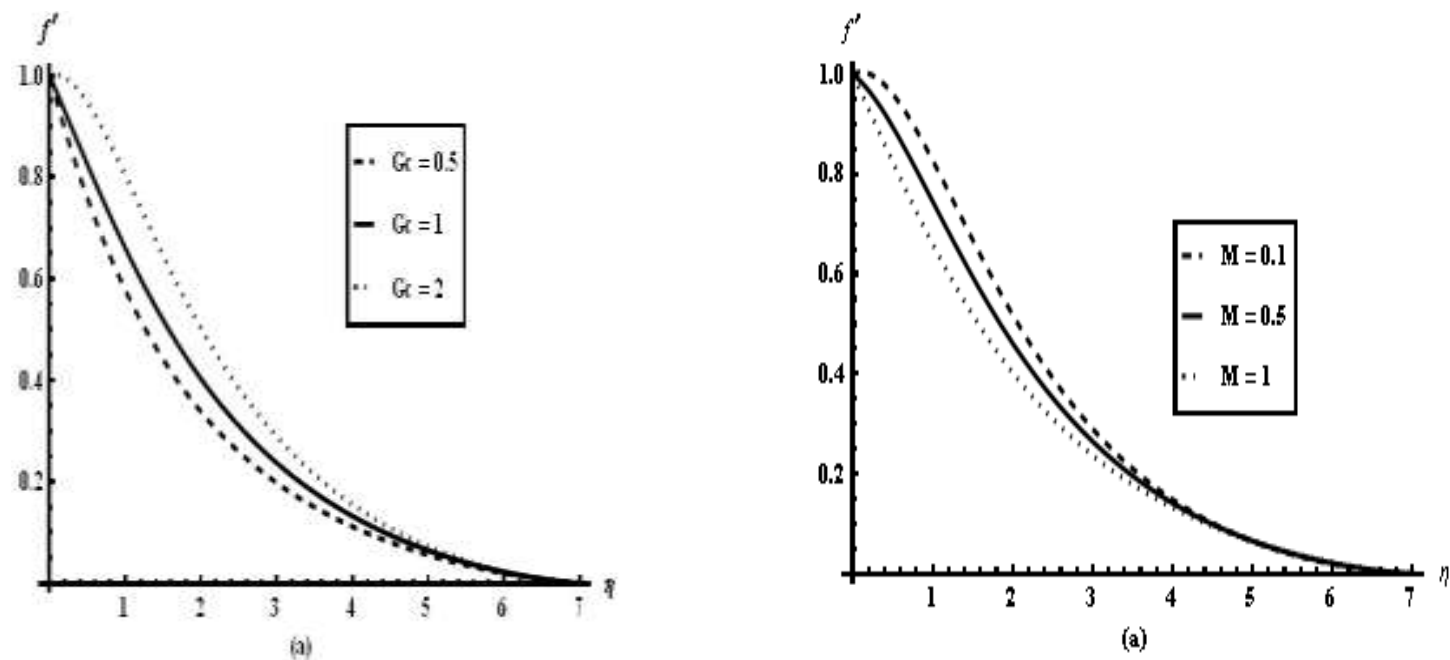

(a)
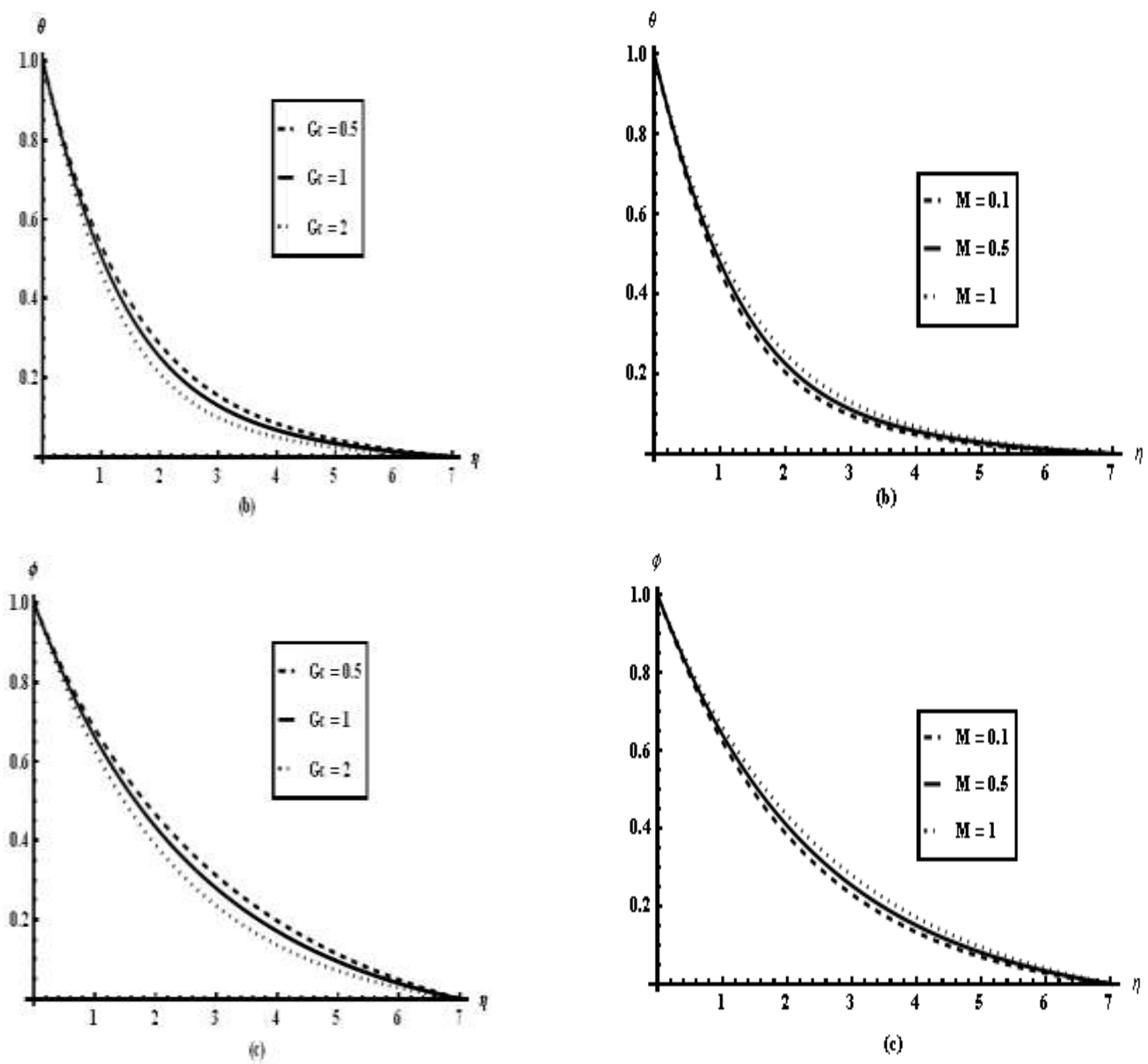

Fig 2. Effect of GC on (a) Velocity,

(b)Temperature and (c) Concentration profiles with $\mathrm{M}=1, \mathrm{~K}=0.1, \operatorname{Pr}=0.71, \mathrm{Du}=1, \mathrm{Sc}=0.22$,

$$
\mathrm{Sr}=0.1, \text { fw }=0.5
$$

Fig 3 . Effect of $M$ on (a) Velocity, (b) Temperature and (c)Concentration profiles with $\mathrm{Gr}=\mathrm{Gc}=\mathrm{K}=0.1, \operatorname{Pr}=0.71, \mathrm{Du}=1$, $\mathrm{Sc}=0.22, \mathrm{Sr}=0.1, \mathrm{fw}=0.5$ 


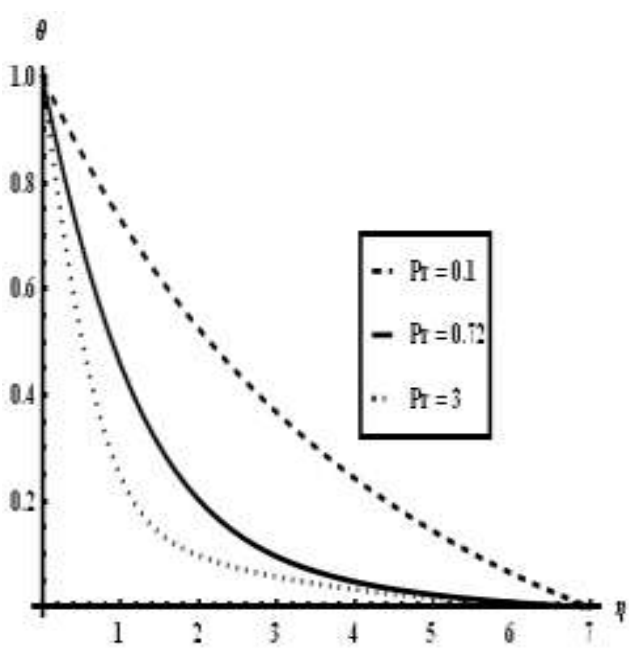

Figure 4: Effect of Pr on temperature profile with $\mathrm{Gr}=\mathrm{Gc}=\mathrm{K}=0.1, \mathrm{M}=1, \mathrm{Du}=1, \mathrm{Sc}=0.22$, $\mathrm{Sr}=0.1, \mathrm{fw}=0.5$

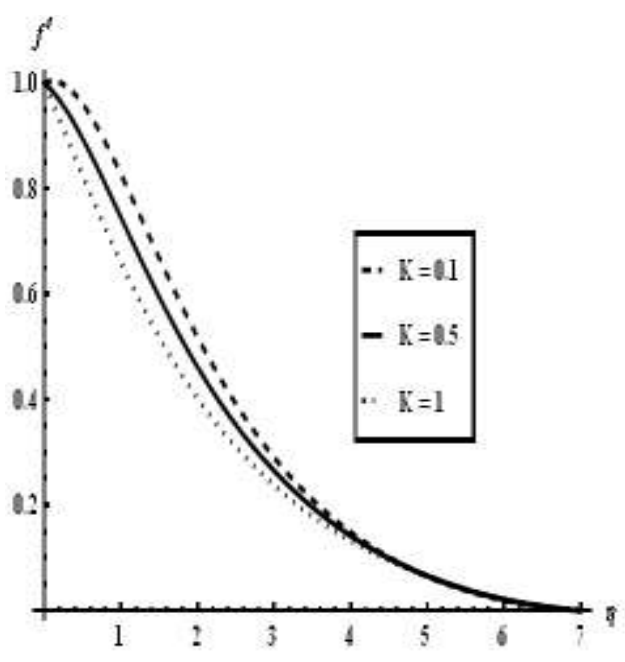

Fig 5. Effect of $K$ on velocity profile with $\mathrm{Gr}=\mathrm{Gc}=0.1, \mathrm{M}=1, \mathrm{Du}=1, \mathrm{Pr}=0.71$,

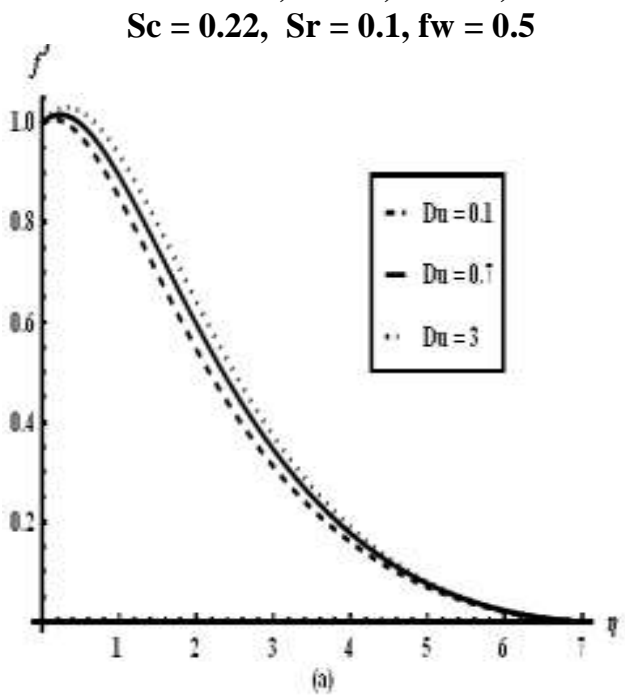

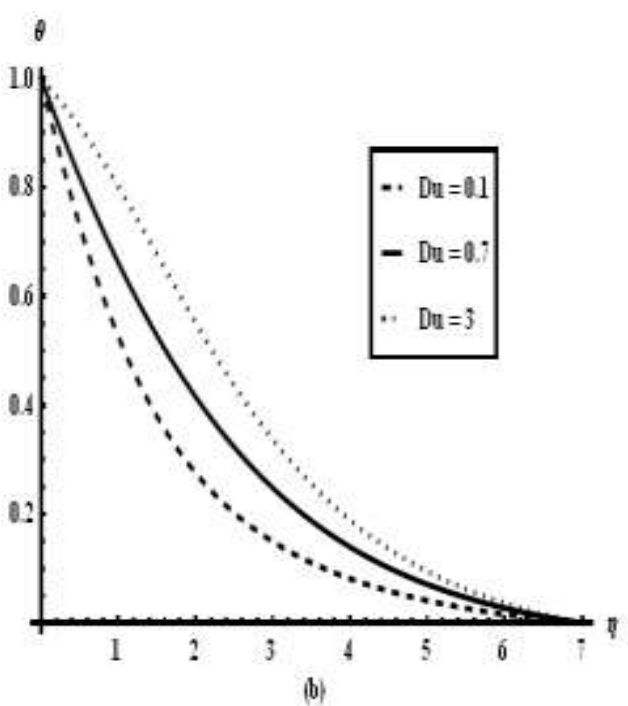

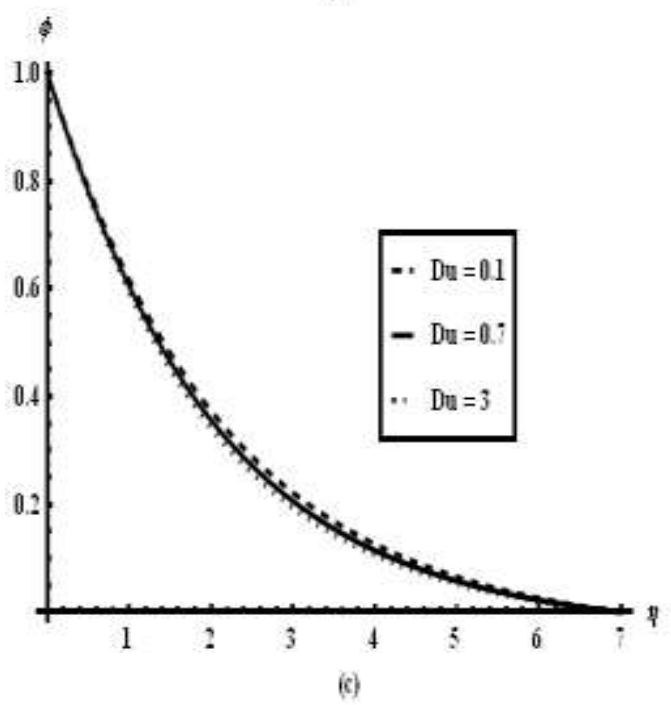

Fig 6. Effect of Dufour number (a) Velocity, (b) Temperature and (c)Concentration profiles with $\mathrm{Gr}=\mathrm{Gc}=\mathrm{K}=\mathbf{0 . 1}, \mathrm{M}=1, \mathrm{Pr}=0.71, \mathrm{Sc}=0.22$, $\mathrm{fw}=0.5$

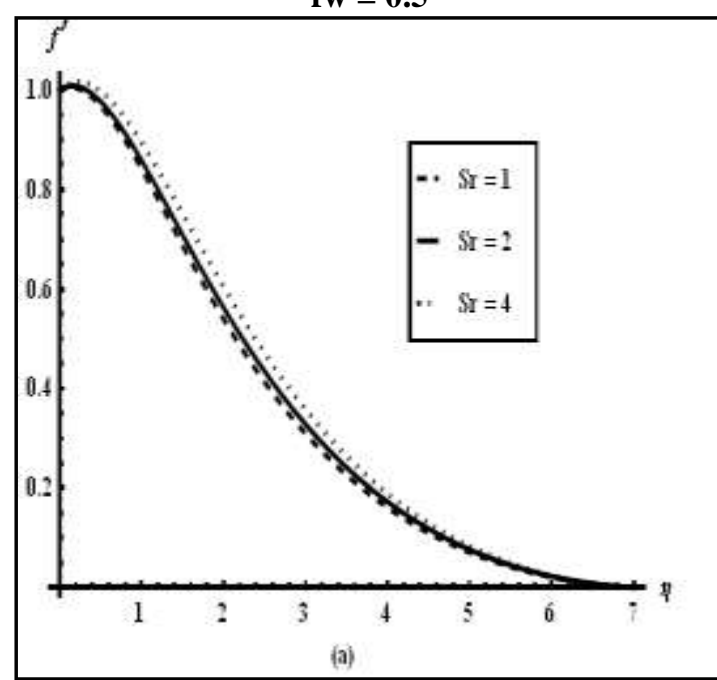



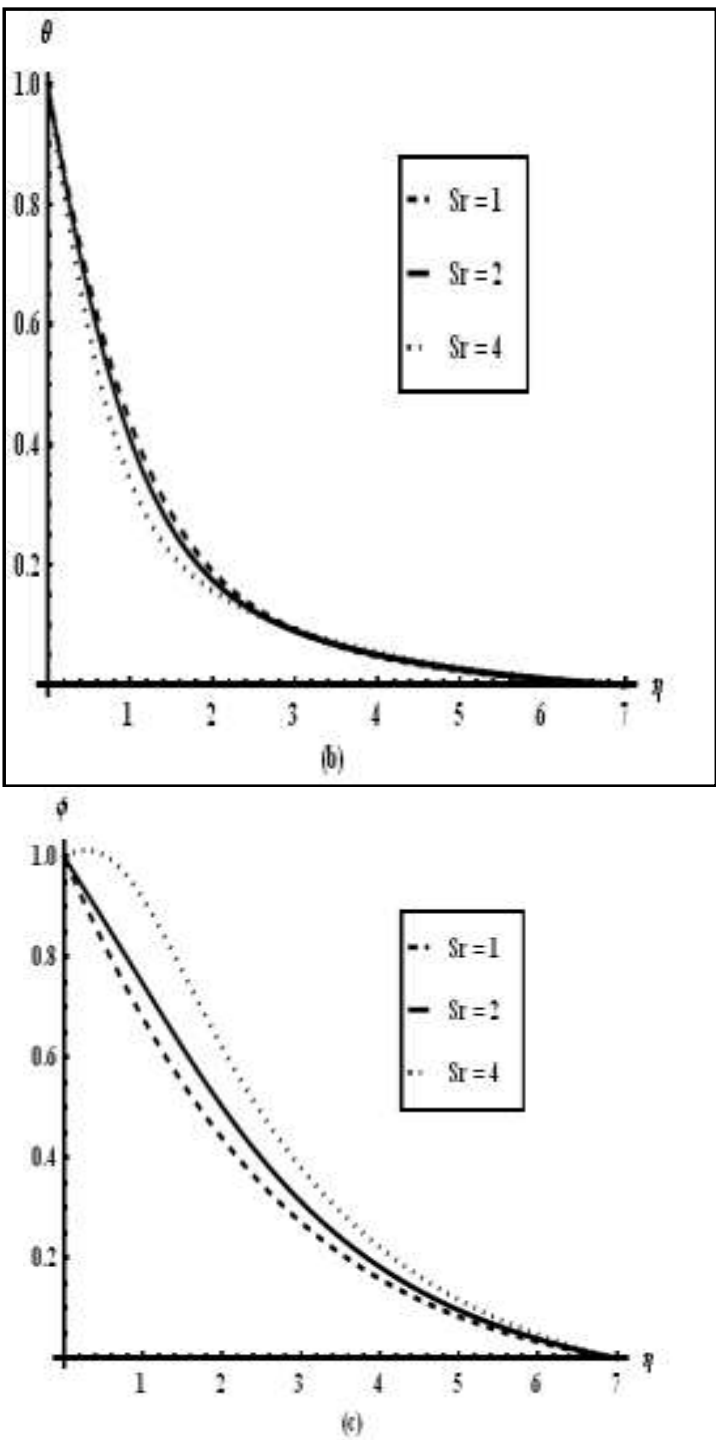

Fig 7. Effect of Soret number on (a) Velocity, (b) Temperature and (c)Concentration profiles with $\mathrm{Gr}=\mathrm{Gc}=\mathrm{K}=0.1, \mathrm{M}=1, \operatorname{Pr}=0.71, \mathrm{Sc}=0.22$,

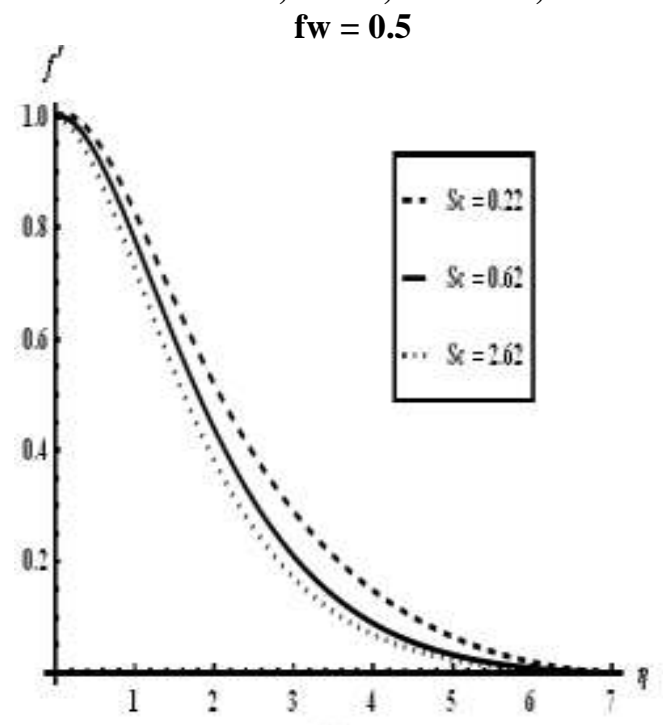

(a)
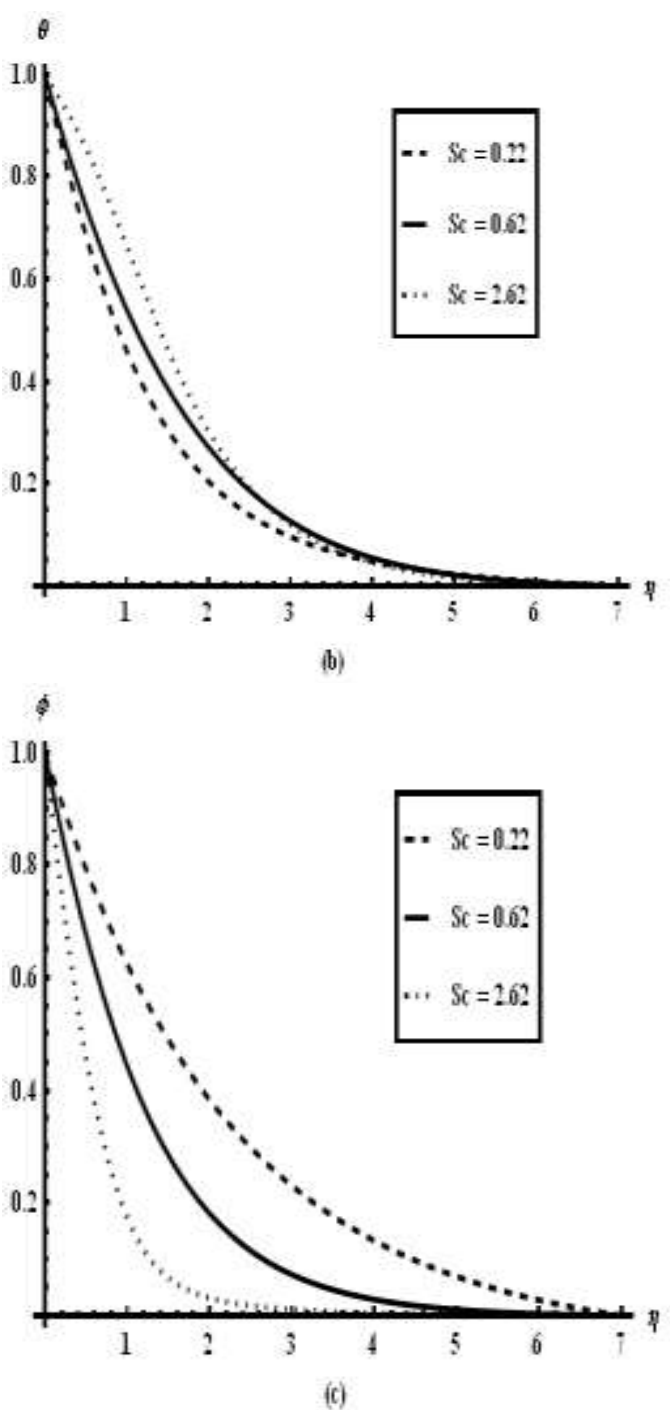

Fig 8. Effect of Schmidth on (a) Velocity, (b)

Temperature and (c)Concentration profiles with $\mathrm{Gr}=\mathrm{Gc}=\mathrm{K}=0.1, \mathrm{M}=1, \operatorname{Pr}=0.71, \mathrm{Du}=1$, $\mathrm{Sr}=0.1$

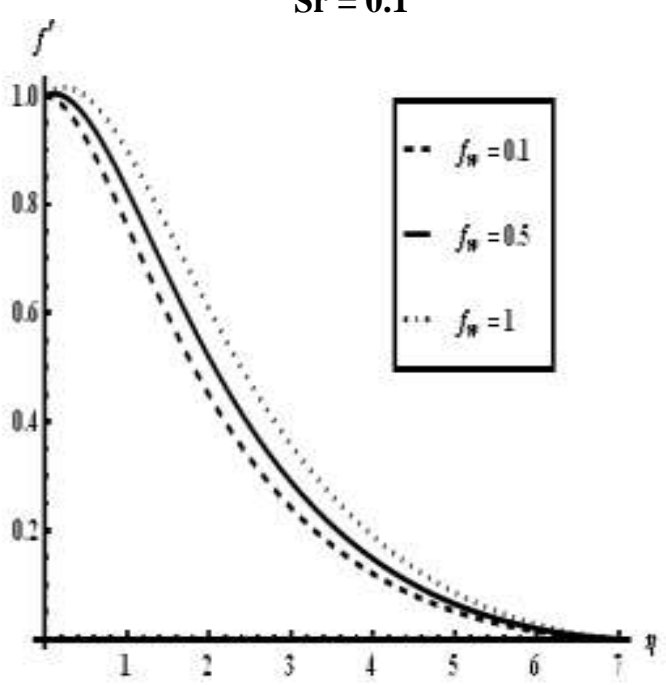

(a) 

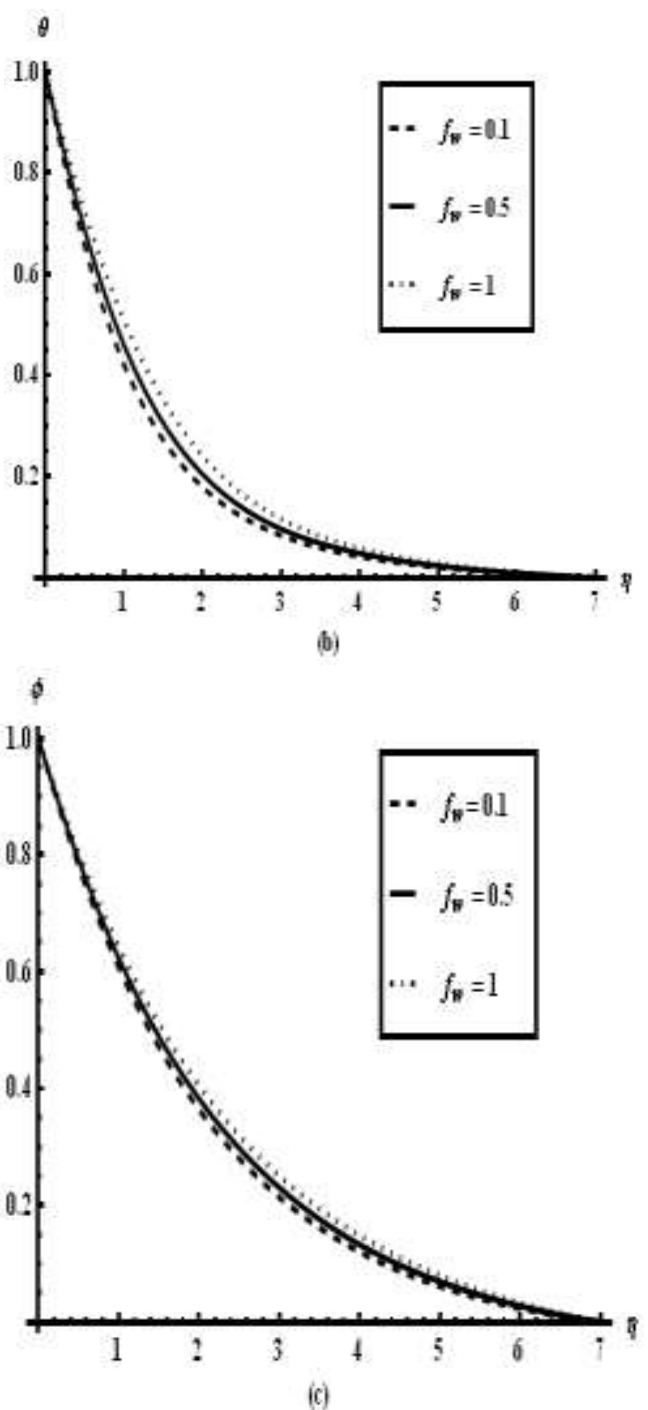

Fig 9. Effect of suction parameter fw on (a) Velocity, (b) Temperature and (c)Concentration profiles with $\mathbf{G r}=\mathbf{G c}=\mathrm{K}=0.1, \mathrm{M}=1, \operatorname{Pr}=0.71$, $\mathrm{Du}=1, \mathrm{Sr}=0.1$

The variation of different values of Dufour Number (Du) on the dimensionless velocity, temperature and concentration distributions is presented in Fig $(6 a-6 c)$. The increase in Dufour number enhances the velocity of the flow which is shown in Fig (6a). It is revealed in Fig (6b) that diffusion thermal affects greatly enfluences the fluid temperature. As the values of the Dufour parameter

\section{REFERENCES}

[1] Abd EL-Naby M. A., El-barbar E. M. E. and Abdelazem N. Y. (2004), An implicit finite difference method to study the effect of radiation on MHD unsteady free convection flow past a semi infinite vertical porous plate but did not take in to account the viscous dissipation, Appl. Math. Comput.151,327 - 346 . increase, the fluid temperature also increases. It is shown in Fig (6c), that the effect of Dufour number is very marginal on concentration profiles.

The effect of the Soret parameter ( $\mathrm{Sr}$ ) on the velocity, temperature and concentration distributions. It is observed in Fig (7a-7c). That the fluid velocity increases with increasing values of the Sr. In the present study it is found that the behaviour of Du and $\mathrm{Sr}$ on temperature and the concentration distributions is opposite.

Velocity and temperature distribution decrease and increase, respectively as (Sc) increases. Fig (8c) shows that the effect of Schmidt number is more on concentration profiles. Fig (9a-9c) illustrates the effect of suction parameter (fw) on the velocity field, temperature and concentration profiles. We see that the velocity, temperature and concentration increases with the increase The influence of Schmidt number Sc is shown in Fig (8a$8 \mathrm{c})$. These figures reveal that the of suction parameter. The influence of $\mathrm{fw}$ on concentration profile is meager. As there is an increse in chemical parameter, it is found that the profile of concentration decreases as depicted in the Fig 10.

\section{CONCLUSIONS}

The numerical study is carried out under the effects of influence of Dufor and Sorect on steady free convection flow past a semi-infinite moving vertical porous plate with suction. The governing partial differential equations are changed into ordinary differential equations by using similarity transformation. These nonlinear, coupled differential equations have been solved numerically by using killer box method. The results are discussed through graphs and tables for different values of parameters modeled in the problem. The results in present attempt reveal that velocity profile decreases with the increase in the magnetic parameter and permeability parameter. It is also noticed in the attempt that the temperature profiles will decrease with an increase in Prandtl number. As Dufour number and Soret Number increases the velocity is also found to increase. It is interesting to note that analysis made on emperature and concentration field is appreciably influenced by the Dufour and Soret effects.

[2] Bejan A. and Khair K.R. (1985), Heat and mass transfer by natural convection in a porous medium, Int. J. Heat Mass Transfer 28, 909-918.

[3] Chamkha A. J. and Khaled (2000.), Hydromagnetic simultaneous heat and mass transfer by mixed convection from a vertical plate embedded in a stratified porous medium with thermaldispersion effects, Heat and Mass Transfer 36, 63-70 
[4] Chamkha A. J. (2003), MHD flow of a numerical of uniformly stretched vertical permeable surface in the presence of heat generation/absorption and a chemical reaction, Int. Comm. Heat Mass transfer 30, $413-422$.

[5] Cookey C. I., Ogulu A. and Omubo-Pepple V. M. (2003), Influence of viscous dissipation and radiation on steady MHD free convection flow past on infinite heated vertical plate in porous medium with time dependent suction, Int. J. Heat Mass Transfer 46, 2305 - 2311.

[6] Eckert E. R. G., Drake, R. M. (1972), Analysis of Heat and Mass Transfer , McGrawHill, NewYork

[7] Hassanien I. A., Bakier A. Y., and Gorla R. S. R. (1998), Effects of thermal dispersion and stratification on non-Darcy mixed convection from a vertical plate in a porous medium, Heat and Mass Transfer 34, 209212.

[8] Huges W.F and Young F.J. (1966), The Electro Magneto Dynamics of fluids. John Wiley and Sons, New York.

[9] Ibrahim F. S., Elaiw A. M. and Bakr A. A. (2008), Effect of the chemical reaction and radiation absorption on the unsteadyMHD free convection flow past a semi - infinite vertical permeable moving plate with heat source and suction, Communications Nonlinear Science Numerical Simulation 13, 1056 - 1066.

[10] Ibrahim S. Y. and Makinde O. D. (2010), Chemically reacting MHD boundary layer flow of heat and mass transfer over a moving vertical plate with suction, Scientific Research and Essays 19, 2875-2882.

[11] Mansour M.A., El-Anssary N.F.and Aly A.M. (2008), Effects of chemical reaction and thermal stratification on MHD free convective heat and mass transfer over a vertical stretching surface embedded in a porous media considering Soret and Dufour numbers, Chemical Engineering Journal $145,340-345$.
[12] Muthucumaraswamy R (2002), Effects of a chemical reaction on a moving isothermal surface with suction, Acta Mechanica 155, 65-72.

[13] Rahman M. M. and M. A. Sattar (2006), Magnetohydrodynamic convective flow of a micropolar fluid past a continuously moving vertical porous plate in the presence of heat generation/absorption, Journal of Heat Transfer 128, 142-152.

[14] Raptis A., Massalas C. and Tzivanidis G., (1982), Hydromagnetic free convection flows through a porous medium between two parallel plates, Physics Letters 90A, 288-289.

[15] Sattar, M.A., Rahman M.M. and Samad M.A. (2001), Similar Solutions of an MHD Free Convection and Mass Transfer Flow with Viscous Dissipation, Dhaka Univ. J. Sci. 49, 67-78.

[16] Seethamahalakshmi, Ramana Reddy G. V. and Prasad B. D. C. N. (2011), Effects of the chemical reaction and radiation absorption on an unsteady MHD convective heat and mass transfer flow past a semi - infinite vertical moving in a porous medium with heat source and suction, IOSR Journal of Engineering, $28-36$.

[17] Sugunamma V., Mohankrishna. P, Sandeep N. and Vidyasagar G.(2011), Radiation effect due to natural convection flow between heated inclined plates under the influence of transverse magnetic field, International journal of mathematical archive, 2336-2346 .

[18] Uwanta I. J.(2012), Effects of chemical reaction and radiation on heat and mass transfer past a semi-infinite vertical porous plate with constant mass flux and dissipation, European Journal of Scientific Research 87, 190-200.

[19] Vidyasagar G., Ramana B. and Bala Anki Reddy P. (2013), Heat and mass transfer effects on mhd boundary layer flow over a moving vertical porous plate, Journal of Global Research in Mathematical Archive, 49-62

International Journal of Engineering Research and Applications (IJERA) is UGC approved Journal with Sl. No. 4525, Journal no. 47088. Indexed in Cross Ref, Index Copernicus (ICV 80.82), NASA, Ads, Researcher Id Thomson Reuters, DOAJ.

G. Balreddy. "Dufour and Soret effect on MHD convective fluid flow in a vertical porous plate with suction ." International Journal of Engineering Research and Applications (IJERA) 7.7 (2017): 05-13. 